\title{
The effect of melatonin on the incidence rates of agitation and delirium in the traumatic brain injury patients
}

\begin{abstract}
Introduction: During the course of healing and increasing the level of consciousness in traumatic brain injury (TBI) after reducing sedatives and dose of analgesics, TBI patients develop some complications, the most common of which are agitation and delirium. Agitation and delirium may deteriorate prognosis and prolong hospital stay. Considering the serious side effects caused by the conventional treatments for agitation and delirium, it is important to prioritize finding safer treatments with lower risks to manage delirium and agitation. Nowadays, melatonin therapy is considered to be effective.
\end{abstract}

Aim: Evaluation of the effect of melatonin on the incidence rate of agitation and delirium in TBI patients.

Materials and methods: In this double-blind clinical trial, 118 patients were studied. The participants were divided into two groups of $\mathrm{A}$ and $\mathrm{B}$ using blocked randomization. Then, group A was given $5 \mathrm{mg} / \mathrm{kg} / \mathrm{h}$ of oral melatonin at 9 p.m. every night, while group B only received synthetic oral placebo. In case of agitation, both groups were prescribed $0.02-0.04 \mathrm{mg} / \mathrm{kg} / \mathrm{h}$ of midazolam to reach Richmond score between -1 and 1. To control pain and reach visual analogue scale (VAS) $<3,0.02-0.04 \mathrm{mg} / \mathrm{kg} / \mathrm{h}$ of intravenous morphine was administered every hour. In case of delirium, based on the confusion assessment method for the intensive care unit (CAM-ICU) criteria, 0.02$0.04 \mathrm{mg} / \mathrm{kg} / \mathrm{h}$ of haloperidol was prescribed. Since the first day of ICU admission until the $10^{\text {th }}$ day of admission, we evaluated and recorded the pain level based on VAS, the administered dose of morphine, the administered dose of midazolam, the incidence rate of delirium based on CAM-ICU and the administered dose of haloperidol every 12 hours. To analyze the data, the two groups were compared based on the frequency of requiring morphine, midazolam and haloperidol, as well as the incidence rates of agitation and delirium by performing Chi-square test in SPSS 11 .

Results: In 118 cases, 97 (82.8\%) were male and 21 (17.8\%) were female, Mean age of 37.79 in melatonin and 36.54 years in placebo group $(\mathrm{P}=0.65)$. The incidence rate of agitation was significantly higher in the placebo group, compared to melatonin group $(\mathrm{P}=0.0001)$. The mean dose of midazolam administration was $7.86 \mathrm{mg} / \mathrm{kg} / \mathrm{h}$ in the melatonin group, while it was $12.79 \mathrm{mg} / \mathrm{kg} / \mathrm{h}$ in the placebo group $(\mathrm{P}=0.0001)$. No significant difference was observed in the mean dose of haloperidol between the melatonin and placebo groups. The mean administered doses melatonin of were 2.64 and $3.38 \mathrm{mg} / \mathrm{kg} / \mathrm{h}$ in the melatonin and placebo groups, respectively ( $\mathrm{P}=0.313)$.

Conclusion: The incidence rate of agitation in patients taking placebo was significantly higher than in those receiving melatonin. In the early ICU admission hours, agitation could be managed and occurred less in patients administered melatonin (group A) rather than those in Group B, which in turn, diminished the administered dose of midazolam $(\mathrm{mg} / \mathrm{kg} / \mathrm{h})$ to control agitation. In the early ICU admission hours, delirium was controlled more effectively in the melatonin group; however, there has been no significant difference between the two groups concerning the mean VAS scores.

Keywords: traumatic brain injury, agitation, delirium, melatonin
Volume 10 Issue 6 - 2018

\author{
Ali Ashraf, Javad Shaarbaf Javan \\ Intensivist, Department of Intensive care, Guilan University of \\ Medical Sciences, Iran
}

Correspondence: Ali Ashraf, Clinical Research, Development Unit of Poursinal Hospital, Guilan University of Medical Sciences, Iran, Tel +989 | | 236 |478, Email draliashvaf@yahoo.fr

Received: January 22, 2018 | Published: November 08, 2018

\section{Introduction}

One of the common causes of intensive care unit (ICU) admission is traumatic brain injury (TBI). During the healing process with increased level of consciousness, TBI patients develop complications with decreased sedation and doses of analgesics, the most common of which are agitation and delirium. ${ }^{1-3}$ The mentioned complications can put patients at risk, deteriorate prognosis and prolong hospitalization. ${ }^{4}$
Agitation is characterized by sudden awakening, irritability and akathisia caused by a sense of discomfort or an internal stimulation and is a behavioural response to physical or mental stimulations such as pain, dyspnea and anxiety. On the other hand, delirium is an acute condition, recognized by impaired consciousness, perceptual alterations, attention deficit and disorganization of behaviour, impaired psychomotor activity and sleep-wake cycle, as well as abnormal awakening. ${ }^{5}$ 
Incidence of agitation and delirium in ICU patients can be associated with some risks. Agitation and delirium can increase the need for sedatives and hypnotics and prolong the duration of ICU stay and the need for ventilator support. Agitation of patients can lead to sudden disconnection of life-sustaining equipment. In addition, they can cause hypoxia, hypotension and barotrauma due to patient-ventilator dyssynchrony. Treatment of agitation without delirium is benzodiazepine, which does not have analgesic effects, but its antianxiety and amnesia properties increase pain tolerance by modifying pain responses. Benzodiazepines can cause hypotension by bolus doses, especially in patients with volume deficit, who cannot tolerate reduction of sympathetic tone. In addition to the reduction of inhibitory effects, these medications can lead to the emergence of contradictory symptoms, such as increase of agitation and aggressive behaviors. The conventional treatment for delirium is the use of antipsychotics. In this regard, non-pharmacological interventions have been proposed, as well. ${ }^{6}$ Antipsychotics are associated with serious complications, such as drowsiness and fall injuries and the incidence of cerebrovascular events is 10 times higher in the first week of treatment with these agents. ${ }^{7}$ Cardiovascular complications, such as increase of QT interval in electrocardiography, have also been reported in this respect. ${ }^{8}$

With regards to the mentioned critical complications, it is recommended to find safer and less risky treatment methods to control delirium and agitation in hospitalized patients. ${ }^{9}$ In this respect, one of the recently proposed treatments is melatonin. ${ }^{10}$ Melatonin, which is known as the night hormone, is chemically a simple amino acid and a small hormone, created by the pineal gland of the brain, retina and red blood cells. This compound is produced in the pineal from tryptophan and serotonin and is secreted in the blood with half-life of 20-30 minutes. Darkness elevated the production of melatonin, whereas light prevents its release. ${ }^{11,12}$ Melatonin is an important hormone and strong antioxidant with a marked role in body reactions, one of the most important of which is regulating the circadian rhythm of humans. Disruption in sleep-wake cycle is one of the factors increasing the incidence of delirium. Therefore, melatonin metabolism alterations can play a significant role in the incidence of delirium. ${ }^{13}$ According to the literature; there is a relationship between delirium and impaired secretion of melatonin. Prescription of exogenous melatonin can prevent the incidence of delirium. ${ }^{14}$ In addition; this medication can contribute to the regulation of sleep cycle of ICU patients and patients with dementia ${ }^{15,16}$

Prophylactic administration of melatonin reduces post-operative delirium. ${ }^{17}$ Given the fact that the similar previous studies were performed on orthopaedic, elderly and dementia patients or those with fractures and there is a scarcity of studies in ICUs or younger TBI patients and considering that delirium and agitation can give rise to serious complications (e.g., prolonged hospitalization), we aimed to evaluate the effect of melatonin on the incidence of delirium and agitation in TBI patients hospitalized in Poursina Hospital.

\section{Materials and methods}

This was a double blind clinical trial, in which the patients, the medication evaluator (subspecialist of ICU) and the nurses of the wards were blinded to the prescribed medication and only the prescriber (the director of the anaesthesia residency program) was informed of the medication. The included population are all the TBI patients admitted to the ICUs of Poursina Hospital, including emergency ICU, neurosurgery ICU and general ICU. The subjects were within the age range of $18-55$ years with $9<$ Glasgow Coma Scale $(\mathrm{GCS}) \geq 13$. The exclusion criteria comprised of discharge before $72 \mathrm{~h}$, death of the patient, decreased level of consciousness (GCS) below nine during the study or surgery (craniotomy) and incidence of seizure during hospital stay. The patients were not incubated (tracheal intubation) and had no history of seizure, addiction to narcotics, mental impairments, use of psychotropic drugs, or organic brain syndrome. All the patients were randomly divided into two groups of A and B using blocked randomization. For group A, $5 \mathrm{mg} / \mathrm{kg} / \mathrm{h}$ of melatonin (Nature Made Co., USA) were orally administered. On the other hand, placebo (Takda Co.) was given to the participants of group B.

Following that, both groups intravenously received $0.02-0.04 \mathrm{mg} /$ $\mathrm{kg} / \mathrm{h}$ of midazolam (Tehran Chemistry Company, Iran) every hour in case of agitation. In addition, in order to reach Richmond score between- 1 and 1 and to control pain, including sympathetic irritability, $0.04-0.02 \mathrm{mg} / \mathrm{kg} / \mathrm{h}$ of morphine (Abureyhan Company, Iran) was prescribed every hour. Further, to achieve a VAS $<3$ and in case of delirium based on the confusion assessment method for the intensive care unit (CAM-ICU) criteria, $0.02-0.04 \mathrm{mg} / \mathrm{kg} / \mathrm{h}$ of haloperidol (Caspian Supply Company, Iran) was prescribed every hour. From the first day of ICU admission until the 10th day of hospitalization, we assessed and recorded the level of pain according to the VAS criteria every 12 hours. In addition, dose of morphine and level of agitation were measured according to the Richmond criteria. Moreover, dose of midazolam and level of delirium were estimated based on CAM-ICU criteria and dose of haloperidol.

Prior to the initiation of the study, the research objectives and methods were explained to the patient companions and written informed consent was obtained from them. The two groups were compared in terms of the frequency of needing morphine, midazolam and haloperidol, as well as the daily incidence of delirium and agitation using Chi-square test in SPSS.

\section{Results}

Out of the 118 subjects, $97(82.8 \%)$ were male and $21(17.8 \%)$ were female and none of the patients was excluded from the study. Mean age of the melatonin receiving group was $37.79 \pm 14.66$ years and mean age of the placebo group was $36.54 \pm 15.98$ years $(\mathrm{P}=0.65)$. Therefore, no significant difference was observed between the study groups regarding age. Figure 1 demonstrates the evaluation of the incidence of agitation (\%) during the study intervals in the melatonin and placebo groups. As can be noted, the incidence rate of agitation was significantly higher in the placebo group, compared to melatonin group $(\mathrm{P}=0.0001)$. The mean dose of midazolam administration was $7.86 \mathrm{mg} / \mathrm{kg} / \mathrm{h}$ in the melatonin group, while it was $12.79 \mathrm{mg} / \mathrm{kg} / \mathrm{h}$ in the placebo group $(\mathrm{P}=0.0001)$. As shown in Figure 2, the incidence rates of delirium at different study intervals (24 and 36 hours) were significantly lower in the melatonin group than the placebo group $(\mathrm{P}=0.008$ and $\mathrm{P}=0.013$, respectively). However, no significant difference was observed in the mean dose of haloperidol between the melatonin and placebo groups. The mean administered doses melatonin of were 2.64 and $3.38 \mathrm{mg} / \mathrm{kg} / \mathrm{h}$ in the melatonin and placebo groups, respectively $(\mathrm{P}=0.313)$. In the current study, no significant difference was observed between the melatonin and placebo groups in terms of pain intensity (based on scale of VAS $>3$ ) at any of the study intervals following ICU admission. Table 1 shows the comparison of the mean ICU admission days in the melatonin (3.84 days) and placebo (4.35 days) groups, demonstrating a significant difference between the groups in this regard. 
Table I Comparison of the mean ICU admission days in the melatonin

\begin{tabular}{llllll}
\hline Statistical estimation & T amount & Standard deviation & Mean (day) & Number & Group \\
\hline $\mathrm{P}=0.000 \mathrm{I}$ & 4.17 & 0.63 & 3.84 & 59 & Melatonin \\
& & 4.35 & 4.35 & 59 & Placebo \\
\hline
\end{tabular}

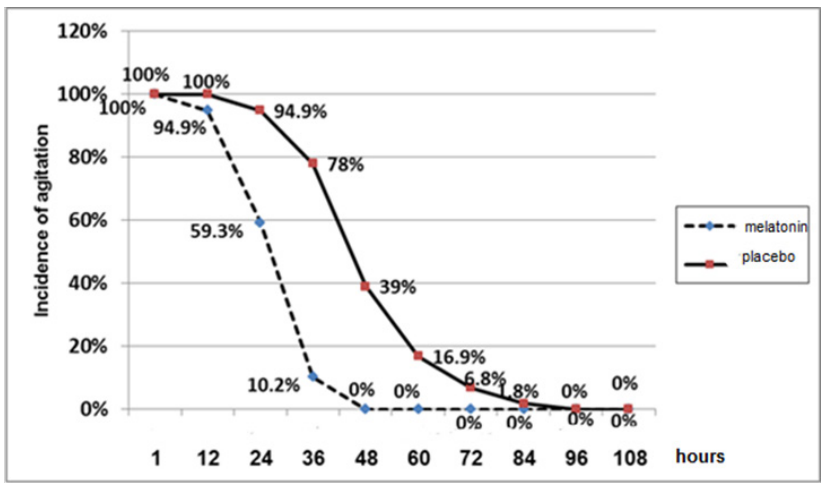

Figure I Incidence of agitation in two groups.

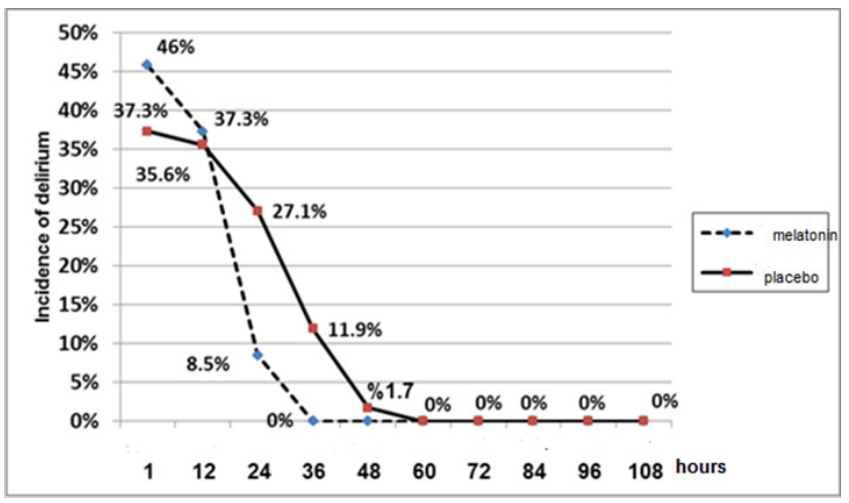

Figure 2 Incidence of delirium in two groups.

\section{Discussion and conclusion}

One of the most common causes of ICU admission is TBI. Patients with TBI are faced with complications in the course of improvement of their consciousness due to decreased sedation and doses of analgesics, the most common of which are agitation and delirium. Agitation and delirium can put patients at risk, deteriorate their prognosis and prolong their hospital stay. Mean age of the melatonin group was $37.79 \pm 14.66$ years, whereas mean age of the placebo group was $36.54 \pm 15.98$ years and most of the patients were young and middle-aged. Given the fact that the majority of the previous studies were conducted on orthopaedic, fracture, dementia, or elderly patients and there are a limited number of studies on ICU patients, one of the strengths of the present study was evaluation of TBI patients and younger patients admitted to ICU and its impact on the duration of hospital stay. In the present study, mean Richmond agitation scale score and the mean midazolam dose administration to achieve a score of -1 to 1 were significantly lower in the melatonin group, compared to the placebo group. In other words, melatonin was able to keep the patients in a more tranquil state. In a study by Bourne et al., the effect of melatonin treatment on improved nocturnal sleep of ICU patients was evaluated in 2008. According to their findings, melatonin increased one hour of nocturnal sleep and decreased AUC BIS.

Moreover, Capuzzo et al. conducted a study in Italy in 2006 to compare the antianxiety effects of melatonin and placebo before surgery. In that study, mean anxiety score was five in melatonin group, which was decreased to three, 90 minutes after receiving the medication. On the other hand, the mean anxiety scores before and after the use of the medication was five and three in the placebo group, respectively. Therefore, both melatonin and placebo equally decreased anxiety. This discrepancy could be due to the short interval between medication administration and evaluation of anxiety. In the current study, agitation was assessed in both melatonin and placebo groups 24, 36, 48 and 60 hours after ICU admission. In the mentioned hours, incidence of agitation was significantly higher in the placebo group, compared to the melatonin group. At other times, there was no statistically significant difference between the study groups in terms of agitation. This indicates that the management and reduction of agitation in early hours of ICU admission was more appropriate in the melatonin group, which led to decreased mean dose of midazolam administration $(\mathrm{mg} / \mathrm{kg} / \mathrm{h})$ to control agitation in the melatonin group.

In a systematic review by Yousaf et al. in Canada in 2009, nine studies demonstrated that melatonin significantly decreased preoperative anxiety, compared to the placebo. However, there is some controversy concerning the analgesic effects of melatonin. In addition, Annemarieke de jonghe et al. conducted a study in the Netherlands in 2011, in which the primary results were indicative of incidence of delirium. They also evaluated the severity, duration and type of delirium, as well as the difference in the time of admission, dose of antipsychotics and/or benzodiazepine during the incidence of delirium and hospital-associated complications. According to the results of mentioned study, the incidence of delirium was decreased by $13 \%$ in the presence of medicinal prophylaxis. However, this reduction was not statistically significant. Similar to the aforementioned study, there was a significant difference in the incidence of delirium (according to CAM-ICU criteria) between the melatonin and placebo groups of the current study at time intervals of 24 and 36 hours after ICU admission. However, no significant difference was observed in this regard at other time intervals. According to the results of the present study, delirium was better controlled in the melatonin group in early hours of ICU admission.

Mean amount of haloperidol consumption $(\mathrm{mg} / \mathrm{kg} / \mathrm{h})$ was nonsignificantly lower in the melatonin group. Furthermore, a significant difference was observed between the study groups regarding the frequency of midazolam administration in the TBI patients admitted to ICU. After discharge from ICU and follow-ups of the patients in wards, no complications due to melatonin were observed.

In addition, no significant difference was found between the melatonin and placebo groups in terms of pain severity in the study intervals and no significant difference was observed in the mean doses 
of administered morphine $(\mathrm{mg} / \mathrm{kg} / \mathrm{h})$ between the groups. In the current study, there was a significant difference between the groups regarding the mean days of ICU stay, such that the mean hospitalization days in the melatonin group was 3.84 days, whereas it was 4.35 days for the placebo group. This could be indicative of antioxidative effects of melatonin in the treatment of TBI patients. Moreover, decreased mean hospitalization days in the melatonin group might be due to reduced dose of antianxiety and antipsychotics used to reduce agitation and delirium.

It is recommended that further studies be conducted using larger sample sizes with separated patients based on the type of brain injury. With regards to the approved antioxidative effects of melatonin in cellular damages, we recommend biochemical evaluation in brain mechanisms and performance of brain cells.

\section{Acknowledgements}

None.

\section{Conflict of interest}

With regards also this title is the final thesis of my anaesthesiology resident Dr Javan.

\section{References}

1. Miller Ronald D, Eriksson Lars I, Fleisher Lee A, et al. Miller `s Anesthesia. Seventh edition, Volume 72, UK: Churchill Livingstone; 2009.

2. Vincent JL, Abraham E, Moore FA, et al. Text book of critical care. $6^{\text {th }}$ edition, Elsevier; 2017:7-10.

3. Hines RL, Marschal KE. Stoelting's Anesthesia and Co-Existing Disease. $6^{\text {th }}$ edition, Elsevier; 2012.

4. Jonghe A, Munster B, Van Oosten H, et al. The effects of melatonin versus placebo on delirium in hip fracture patients: study protocol of a randomised, placebo-controlled, double blind trial. BMC Geriatr. $2011 ; 11: 34$
5. Sultan SS. Assessment of role of perioperative melatonin in prevention and treatment of postoperative delirium after hip arthroplasty under spinal anesthesia in the elderly. Saudi J Anaesth. 2010;4:169-73.

6. Siddiqi N, Stockdale R, Britton AM, et al. Interventions for preventing delirium in hospitalised patients. Cochrane Database Syst Rev. 2007;18(2):CD005563.

7. Kleijer BC, Marum RJ, Egberts AC, et al. Risk of cerebrovascular events in elderly users of antipsychotics. J Psychopharmacol. 2009;23(8):909-914.

8. Stollberger C, Huber JO, Finsterer J. Antipsychotic drugs and QT prolongation. Int Clin Psychopharmacol. 2005;20(5):243-251.

9. Jonghe A, Korevaar JC, Munster BC, et al. Effectiveness of melatonin treatment on circadian rhythm disturbances in dementia.Are there implication for delirium? A systematic review. Int J Geriat Psychiatry. 2010;25:1201-1208.

10. Seifman MA, Adamides AA, Nguyen PN, et al. Endgenous melatonin increases in cerebrospinal fluid of patients after sever traumatic brain injury and correlates with oxidative stress and metabolic disarray. $J$ Cereb Blood Flow Metab. 2008;28(4):684-696.

11. Brzezinski A. Melatonin in humans. N Engl J Med. 1997;336:186-195.

12. Muller E. Melatonin hormone.

13. Lewis MC, Barnett SR. Postoperative delirium: the tryptophan dysregulation model. Med Hypotheses. 2004;63:402-406.

14. Hanania M, Kitain E. Melatonin for Treatment and Prevention of Postoperative Delirium. Anesth Analg. 2002;94(2):338-339.

15. Shilo L, Dagan Y, Smorjik Y, et al. Effect of melatonin on sleep quality of COPD intensive care patients: a pilot study. Chronobiol Int. 2000;17:71-76

16. Bourne RS, Mills GH, Minelli C. Mellatonin therapy to improve nocturnal sleep in critically ill patients:encouraging results from a small randomsed controlled trial. Crit Care. 2008;12(2):R52.

17. Al Aama T, Brymer C, Woolmore GSM, et al. Melatonin decreases delirium in elderly patients: a randomized, placebo-controlled trial. Int $J$ Geriatr Psychiatry. 2011;26(7):687-694. 